\title{
Baby Universes and String Theory
}

\section{Citation}

Dijkgraaf, Robbert, Rajesh Gopakumar, Hirosi Ooguri, and Cumrun Vafa. 2006. “Baby Universes in String Theory." Physical Review D 73 (6). https://doi.org/10.1103/physrevd.73.066002.

\section{Permanent link}

http://nrs.harvard.edu/urn-3:HUL.InstRepos:41385098

\section{Terms of Use}

This article was downloaded from Harvard University's DASH repository, and is made available under the terms and conditions applicable to Other Posted Material, as set forth at http:// nrs.harvard.edu/urn-3:HUL.InstRepos:dash.current.terms-of-use\#LAA

\section{Share Your Story}

The Harvard community has made this article openly available.

Please share how this access benefits you. Submit a story.

Accessibility 


\title{
Baby universes in string theory
}

\author{
Robbert Dijkgraaf, ${ }^{1}$ Rajesh Gopakumar, ${ }^{2}$ Hirosi Ooguri, ${ }^{3}$ and Cumrun Vafa ${ }^{4}$ \\ ${ }^{1}$ Institute for Theoretical Physics \& KdV Institute for Mathematics, University of Amsterdam, \\ Valckenierstraat 65, 1018 XE Amsterdam, The Netherlands \\ ${ }^{2}$ Harish-Chandra Research Institute, Chhatnag Road, Jhusi, Allahabad 211019, India \\ ${ }^{3}$ California Institute for Technology, 452-48, Pasadena, California 91125, USA \\ ${ }^{4}$ Jefferson Physical Laboratory, Harvard University, Cambridge, Massachusetts 02138, USA
}

(Received 7 October 2005; published 10 March 2006)

\begin{abstract}
We argue that the holographic description of four-dimensional Bogomol'nyi-Prasad-Sommerfield black holes naturally includes multicenter solutions. This suggests that the holographic dual to the gauge theory is not a single $\mathrm{AdS}_{2} \times S^{2}$ but a coherent ensemble of them. We verify this in a particular class of examples, where the two-dimensional Yang-Mills theory gives a holographic description of the black holes obtained by branes wrapping Calabi-Yau cycles. Using the free fermionic formulation, we show that $O\left(e^{-N}\right)$ nonperturbative effects entangle the two Fermi surfaces. In an Euclidean description, the wave function of the multicenter black holes gets mapped to the Hartle-Hawking wave function of baby universes. This provides a concrete realization, within string theory, of effects that can be interpreted as the creation of baby universes. We find that, at least in the case we study, the baby universes do not lead to a loss of quantum coherence, in accord with general arguments.
\end{abstract}

DOI: 10.1103/PhysRevD.73.066002

PACS numbers: 11.25.Mj, 04.70.Dy

\section{INTRODUCTION}

The study of quantum aspects of black holes has led to important progress in a deeper understanding of quantum gravity. One basic notion is that of black hole entropy, which was predicted by Bekenstein [1] and Hawking [2], through semiclassical reasoning, to be one quarter of the area of the horizon in Planck units. More recently it was shown in the context of string theory [3] that for special classes of black holes the microstates of the black hole consist of bound states of suitable configuration of branes (see [4-6] for reviews of this subject). In particular it was found that the semiclassical reasoning of Hawking agrees with the leading large charge entropy of black hole microstates constructed within string theory.

However, in string theory one can go further and compute, in addition, the subleading corrections to the black hole entropy. More specifically, in the context of certain extremal black holes obtained in compactifications of type II strings on Calabi-Yau three-folds these corrections are captured by topological string amplitudes [7]. These results recently have led to a concrete formulation of the quantum corrected black hole entropy to all orders in string perturbation theory [8]. This states that the partition function of a statistical ensemble of black hole states $Z_{\mathrm{BH}}$ is given by the norm-squared of the topological string wave function $^{1}$ on the corresponding Calabi-Yau three-fold:

\footnotetext{
${ }^{1}$ In this paper, we refer to the topological string partition function $\psi_{\text {top }}=\exp \left(\sum_{g} F_{g}\right)$ as a wave function following the interpretation $[9,10]$ of the holomorphic anomaly equations [11] for $\psi_{\text {top }}$ as representing the background independence of the geometric quantization of the tangent space to the moduli space of the Calabi-Yau three-fold.
}

$$
Z_{\mathrm{BH}}=\left|\psi_{\mathrm{top}}\right|^{2}
$$

Since in this relation the string coupling constant is inversely proportional to the charge, large charge black holes get mapped to the weak coupling limit of topological strings.

The appearance of the notion of a wave function in the above formula, at first sight, sounds surprising since we are dealing with a partition function. This was explained in [12], where it was identified as a Hartle-Hawking wave function associated to a radial quantization, as contrasted to the usual temporal quantization, of the Euclidean black hole geometry. In particular, as was observed in [12], the Bogomol'nyi-Prasad-Sommerfield monopoles (BPS) minisuperspace Hilbert space $\mathcal{H}_{M}$ (for type IIB compactifications) corresponds to the geometric quantization of the phase space $H^{3}(M)$, where $M$ is the corresponding Calabi-Yau three-manifold. Moreover, fixing the electric/ magnetic fluxes $(Q, P)$ leads to a distinguished state in this Hilbert space

$$
|Q, P\rangle=e^{(i \pi) / 2\left(Q_{I} X^{I}-P^{I} F_{I}\right)}|0,0\rangle \in \mathcal{H}_{M},
$$

where $X^{I}, F_{I}$ are suitable (canonically conjugate) operators, with the property that the black hole state degeneracy $\Omega(Q, P)$ corresponds to

$$
\Omega(Q, P)=\langle Q, P \mid Q, P\rangle=\int \prod_{I} d \phi^{I}\left|\psi_{(Q, P)}(\phi)\right|^{2}
$$

In the real polarization on $H^{3}(M)$, this wave function is related to the topological string partition function via 


$$
\begin{aligned}
\psi_{(Q, P)}(\phi) & =e^{-(1 / 2) Q_{I} \phi^{I}} \psi_{\mathrm{top}}\left(P+\frac{i}{\pi} \phi\right) \\
& =e^{-[(i \pi) / 4] Q_{I} P^{I}-(1 / 2) Q_{I} \phi^{I}-\pi i P^{I}\left[\partial /\left(\partial \phi^{I}\right)\right]} \psi_{\mathrm{top}}\left(\frac{i}{\pi} \phi\right) .
\end{aligned}
$$

Some aspects of the background independence of the black hole entropy and its relation to this Hilbert space has been discussed in [13] .

The prediction (1.1) has been verified in a number of examples [14-19] In particular, in the context of a $T^{2}$ embedded in the Calabi-Yau manifold, it was shown in [14] that the bound state of D4-, D2-, and D0-branes maps to the partition function of $U(N)$ two-dimensional YangMills (YM) theory on $T^{2}$. Here the number of D4-branes corresponds to the rank $N$ of the gauge group and the chemical potentials for D2- and D0-branes can be identified with some combination of the theta angle and the gauge coupling of the Yang-Mills theory. The fact that in this case the Yang-Mills partition function takes the form of the norm-squared of a holomorphic object follows from the results in [20] where the 't Hooft large $N$ limit of YangMills theory was studied.

However, as was noted in [14], there are additional nonperturbative corrections (behaving like $e^{-N}$ ) to the large $N$ limit which destroy the holomorphic factorization property (1.1). The lack of exact factorization is best understood in the free nonrelativistic fermion formulation of Yang-Mills theory where the two Fermi surfaces are entangled at finite $N$. Our main goal in this paper is to study these corrections and interpret their physical meaning in the dual superstring theory. ${ }^{2}$

What we find is that the correction terms to the large $N$ limit of the D-brane gauge theory can be interpreted as arising from multicenter black holes [22], a special case of which correspond to the Brill instantons [23]. This, in particular, leads to the statement that, while the perturbative $1 / N$ expansion holographically describes a single black hole, via its nonperturbative $\mathcal{O}\left(e^{-N}\right)$ effects, the gauge theory is actually dual to a coherent ensemble of black holes. This is an interesting twist to the notion of holography and may lead to a resolution to the puzzles raised in [24] in the context of $\mathrm{AdS}_{2}$ holography. From the viewpoint of radial quantization [12], our conclusion is therefore that the Hartle-Hawking wave function is not a single universe wave function but rather the wave function for an ensemble of baby universes.

In particular, in this language we find that the suitable wave function belongs to the "third quantized" Hilbert

\footnotetext{
${ }^{2}$ These nonperturbative effects also explain the origin of apparent discrepancies in some of the examples studied in [21], where computations are done in the strong coupling regime, $g_{s} \gg 1$. Because of the nonperturbative corrections, we expect $O(1)$ corrections to the perturbative formula (1.1) in this regime and thus we find the question is reversed: Why did some of the examples in [21] work at all?
}

space, where we have one Hilbert space per baby universe. ${ }^{3}$ We find that in order to capture nonperturbative corrections we need to consider the total Hilbert space

$$
\mathcal{H}=\bigoplus_{n \geq 0} \mathcal{H}_{M}^{\otimes n}
$$

We find that, if we fix the total flux corresponding to electric/magnetic charges $(Q, P)$, the complete quantum state $\left|\psi_{\text {total }}(Q, P)\right\rangle$ receives contribution from the HartleHawking wave function of an arbitrary number of baby universes and belongs to $\oplus_{n} \mathcal{H}_{M}^{\otimes n}$. It takes a particularly simple form. The $n$ universe state is essentially given by

$$
\left|\psi_{n}(Q, P)\right\rangle=\sum_{\substack{Q_{1}+\ldots+Q_{n}=Q, P_{1}+\ldots+P_{n}=P}}\left|Q_{1}, P_{1} ; Q_{2}, P_{2} ; \cdots ; Q_{n}, P_{n}\right\rangle
$$

with a suitable range of sum over charges (and modulo some positivity constraint) and where

$$
\begin{aligned}
\left|Q_{1}, P_{1} ; Q_{2}, P_{2} ; \cdots ; Q_{n}, P_{n}\right\rangle= & \left|Q_{1}, P_{1}\right\rangle \otimes\left|Q_{2}, P_{2}\right\rangle \otimes \cdots \\
& \otimes\left|Q_{n}, P_{n}\right\rangle .
\end{aligned}
$$

This multiuniverse wave function is related to the net black hole entropy $\Omega(Q, P)$ by the formula

$$
\sum_{n=1}^{\infty}(-1)^{n-1} C_{n-1}\left\langle\psi_{n}(Q, P) \mid \psi_{n}(Q, P)\right\rangle=\Omega(Q, P),
$$

where $C_{n}$, the $n$th Catalan number, counts the number of planar binary trees with $(n+1)$ branches, i.e. the number of distinct ways the baby universes can be produced. The sign factor $(-1)^{n-1}$ is perhaps unexpected. This extends the analysis of [12] to the wave function of universes which are spatially disconnected.

There is a natural interpretation of this simple factorization structure from the viewpoint of the dual gravity solutions, which turn out to be multicenter black hole solutions [22]. Each of the component wave functions is associated to the near-horizon geometry of the corresponding black hole. The structure we have found for the multibaby universe wave function suggests that there is no loss of quantum coherence, in line with the predictions of Coleman [26]. Essentially, this is because if we measure the fluxes through one baby universe $\left(Q_{i}, P_{i}\right)$, the corresponding wave function is fixed to be $\left|Q_{i}, P_{i}\right\rangle$ and is independent of the other fluxes $\left(Q_{r}, P_{r}\right)_{r \neq i}$ or the degrees of freedom on the other universes.

The organization of this paper is as follows: In Sec. II we review the two-dimensional Yang-Mills partition function and its large $N$ limit. We also review its relation to black hole entropy. In Sec. III we consider $\mathcal{O}\left(e^{-N}\right)$ corrections to the large $N$ limit of Yang-Mills theory and their relation to BPS partition functions of D-brane systems. We give an interpretation of these effects in terms of multiple wave

\footnotetext{
${ }^{3}$ String theoretic realization of baby universes has recently been discussed in a different context in [25].
} 
functions. In Sec. IV we review the relevant multicenter gravity solutions. Finally, in Sec. V we interpret the nonperturbative large $N$ corrections in the context of the holographically dual gravity solutions.

\section{TWO-DIMENSIONAL YANG-MILLS AND TOPOLOGICAL STRING THEORY}

\section{A. Topological strings on local $T^{2}$}

Let us briefly review the setup of [14] that relates topological strings, two-dimensional Yang-Mills theory, and black hole degeneracies. The starting point is a noncompact Calabi-Yau manifold $M$ given by the total space of the following rank two vector bundle over a two-torus

$$
\mathcal{O}(m) \oplus \mathcal{O}(-m) \rightarrow T^{2} .
$$

Here $m$ is a given integer. This noncompact space can be considered as the local neighborhood of an elliptic curve embedded in a compact Calabi-Yau manifold.

We will consider the A-model topological string on the geometry (2.1). The string partition function $\psi_{\text {top }}\left(t, g_{s}\right)$ will depend on the cohomology class $t \in H^{1,1}\left(T^{2}\right)$ of the complexified Kähler form $k$ on $T^{2}$ and the string coupling constant $g_{s}$. It has a perturbative expansion of the form

$$
\psi_{\text {top }}\left(t, g_{s}\right)=\exp \sum_{g \geq 0} g_{s}^{2 g-2} F_{g}(t)
$$

where $F_{g}(t)$ is the contribution at genus $g$ in string perturbation theory. The stringy contributions to these perturbative terms can be viewed as generated by world-sheet instanton effects. If the Gromov-Witten invariant $N_{d, g}$ denotes the "number" of instantons of degree $d$ and genus $g$ (it is in general a rational number), then

$$
F_{g}(t)=\sum_{d \geq 0} N_{g, d} e^{-d t} .
$$

These contributions are only nonzero for $g \geq 1$. In addition to these world-sheet instanton effects, there are classical contributions at genus zero and one, given by certain intersection numbers. In general, for a noncompact target space these are a bit ambiguous, but in the case of $M$ these can be computed to be [14]

$$
F_{0}^{\mathrm{cl}}(t)=-\frac{1}{6} \frac{t^{3}}{m^{2}}, \quad F_{1}^{\mathrm{cl}}(t)=\frac{1}{24} t .
$$

After including these classical parts, the net partition functions $F_{g}(t)$ are quasimodular forms of weight $(6 g-6)$ under the usual action of $S L(2, \mathbf{Z})$ on $\tau=i t / 2 \pi$. For example, [27,28]

$$
\begin{aligned}
& F_{1}(t)=-\log \eta, \\
& F_{2}(t)=\frac{1}{103680}\left(10 E_{2}^{3}-6 E_{2} E_{4}-4 E_{6}\right),
\end{aligned}
$$

with $\eta$ the Dedekind eta function and $E_{n}$ the Eisenstein series, being (quasi)modular functions of weight $n$. This modularity can be understood from applying mirror symmetry to the $T^{2}$ that turns $\tau$ into the complex modulus of the dual elliptic curve [29] and has been rigorously proven in [30]. Furthermore, if a suitable antiholomorphic dependence is added, in accordance with the holomorphic anomaly of the string partition function [31], full modularity is restored [32].

\section{B. D-branes and two-dimensional Yang-Mills theory}

We will now consider a type IIA compactification on the Calabi-Yau space $M$. We can wrap $N$ D4-branes, which we take to cover the base $T^{2}$ and one of the two complex fiber directions. (This breaks the symmetry $m \rightarrow-m$.) This will give us a $4+1$-dimensional supersymmetric $U(N)$ gauge theory, that describes a point particle in the four noncompact dimensions. We can further consider bound states with $N_{2}$ D2-branes, that wrap the $T^{2}$, and $N_{0}$ D0-branes. These lower-dimensional branes will be represented by the Chern classes $c_{1}(\mathcal{E})$ and $c_{2}(\mathcal{E})$ that capture the topology of the gauge bundle $\mathcal{E}$ of the D4-brane.

After taking into account the backreaction of the supergravity, this collection of D-branes will manifest itself as a charged four-dimensional black hole. Since we do not have any D6-brane charge, the electric and magnetic charges are, respectively, given by

$$
Q=\left(N_{2}, N_{0}\right), \quad P=(N, 0) .
$$

The black hole partition function that counts the number of BPS states can be identified with an index of the corresponding gauge theory. In fact, as explained in [8] and as we will review at greater length in Sec. IV, within the string theory context it is more natural to compute this partition function in a mixed ensemble, where we fix the magnetic charges $P$ and introduce chemical potentials for the electric charges $Q$. In this case this means that we fix the rank $N$ of the gauge group and sum over the different topologies of the gauge bundle. The chemical potentials for D0 and D2 branes can be identified with $\frac{4 \pi^{2}}{g_{s}}$ and $\frac{2 \pi \theta}{g_{s}}$, respectively, where the angle $\theta$ is the coefficient of the $\operatorname{Tr} F \wedge k$ term in the four-dimensional action.

So the gauge theory/black hole partition function takes the form

$$
\begin{aligned}
Z_{N}\left(g_{s}, \theta\right)= & \sum_{N_{2}, N_{0} \geq 0} \Omega\left(N ; N_{2}, N_{0}\right) \\
& \cdot \exp \left(-\frac{4 \pi^{2} N_{0}}{g_{s}}-\frac{2 \pi N_{2} \theta}{g_{s}}\right) .
\end{aligned}
$$

Here $\Omega\left(N ; N_{2}, N_{0}\right)$ denotes the index of BPS bound states with the given charges. In order to relate this black hole partition function with the closed topological string partition function as in (1.1), one needs to make the following 
identification of the closed and open string moduli [14]

$$
t=\frac{1}{2} g_{s} m N+i \theta .
$$

As is explained in [14], this particular D4-brane system can be further simplified and in fact be identified with a two-dimensional bosonic Yang-Mills theory on the compact base $T^{2}$. The action of this model is best written in Hamiltonian form with $F$ the field strength of the gauge field $A$, together with an additional adjoint scalar $\Phi$ (that is the dual momentum to $A$ )

$$
S=\frac{1}{g_{s}} \int_{T^{2}} \operatorname{Tr}\left(\Phi F+\theta \Phi+\frac{1}{2} m \Phi^{2}\right) .
$$

Here we have set the area of the two-torus to one. Then we can identify the gauge coupling with

$$
g_{\mathrm{YM}}^{2}=m \cdot g_{s} .
$$

We can also absorb the factor $m$ by redefining $g_{s} \rightarrow m g_{s}$, at least if $m$ is nonzero. ${ }^{4}$

Since the solution of two-dimensional Yang-Mills theory on a general Riemann surface is well known [33-37], these identifications give the following exact expression for the black hole partition function expressed as a sum over all irreducible representations $R$ of $U(N)$

$$
Z_{N}=\sum_{R} e^{-(1 / 2) g_{s} C_{2}(R)+i \theta C_{1}(R)} .
$$

Here $C_{1}(R)$ and $C_{2}(R)$ are the first and second Casimir invariants of the representation $R$.

\section{Free fermion system}

Two-dimensional Yang-Mills theory on a torus has an elegant reformulation in terms of a system of $N$ nonrelativistic free fermions moving on a circle [38,39]. With natural antiperiodic boundary conditions, these fermions have half-integer quantized momenta

$$
p \in \mathbf{Z}+\frac{1}{2} \text {. }
$$

In the fermion correspondence, a YM state labeled by a definite irreducible representation $R$ of $U(N)$ is given by filling some particular levels $p_{1}, \ldots, p_{N}$. The ground state, given by the trivial representation, is obtained by filling the states from $p=-\frac{1}{2} N$ to $p=+\frac{1}{2} N$; see Fig. 1(a). The nontrivial representations correspond to the excitations of the top and the bottom Fermi levels, as depicted in Fig. 1(b).

The Casimirs of the Yang-Mills representations can be expressed as the total energy and momentum of this $N$-fermion state

$$
\frac{1}{2} C_{2}(R)=E-E_{0}, \quad C_{1}(R)=P,
$$

\footnotetext{
${ }^{4}$ Note that this chooses the sign of the string coupling $g_{s}$. Changing $m \rightarrow-m$, which corresponds to picking the other line bundle to wrap the D4-brane, can be compensated by $g_{s} \rightarrow-g_{s}$.
}

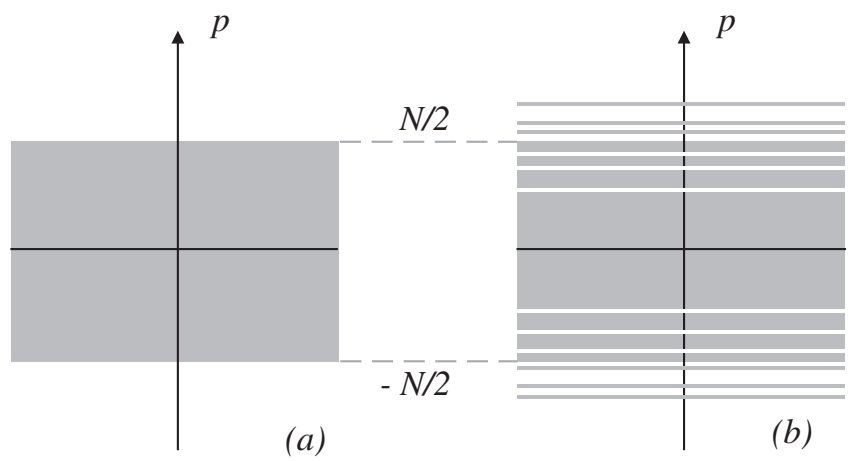

FIG. 1. The spectrum of two-dimensional Yang-Mills is described by a system of $N$ free fermions. Depicted are the ground state (a) and small excitations above the ground state (b), given by fluctuations of the two Fermi surfaces at $p= \pm N / 2$.

with

$$
E=\sum_{i=1}^{N} \frac{1}{2} p_{i}^{2}, \quad P=\sum_{i=1}^{N} p_{i} .
$$

Here $E_{0}$ is the ground state energy

$$
E_{0}=\frac{1}{24}\left(N^{3}-N\right) \text {. }
$$

It is convenient to add this overall energy shift to the YangMills theory and define

$$
Z_{N}=\sum_{\text {fermions }} e^{-g_{s} E+i \theta P} .
$$

This shift $\frac{1}{2} C_{2} \rightarrow \frac{1}{2} C_{2}+E_{0}$ is also natural from the string perspective, since it produces exactly the classical contributions to the topological partition function [14]

$$
E_{0}=-F_{\text {top }}^{\mathrm{cl}}(t)-\bar{F}_{\text {top }}^{\mathrm{cl}}(\bar{t}),
$$

where

$$
F_{\mathrm{top}}^{\mathrm{cl}}(t)=\frac{1}{g_{s}^{2}} F_{0}^{\mathrm{cl}}(t)+F_{1}^{\mathrm{cl}}(t)=-\frac{t^{3}}{6 g_{s}^{2}}+\frac{t}{24},
$$

with $t=\frac{1}{2} g_{s} N+i \theta$.

\section{The large $N$ limit and chiral fermions}

As discovered by Gross and Taylor [20], twodimensional Yang-Mills theory simplifies considerably in the large $N$ limit, defined as

$$
N \rightarrow \infty, \quad g_{s} \rightarrow 0, \quad \text { with } g_{s} N \text { fixed. }
$$

The simplification is most easily understood in the fermionic reformulation. Here the dynamics of the large $N$ limit is in good approximation described by independent fluctuations of the two Fermi surfaces that will be separated by a distance $N$; see Fig. 2. In fact, in the $U(N)$ gauge theory [as contrasted with the $S U(N)$ theory] the Fermi levels can be in general position at $p=N_{+}$and $p=-N_{-}$(up to a shift by $\frac{1}{2}$ that we will ignore) as long as 


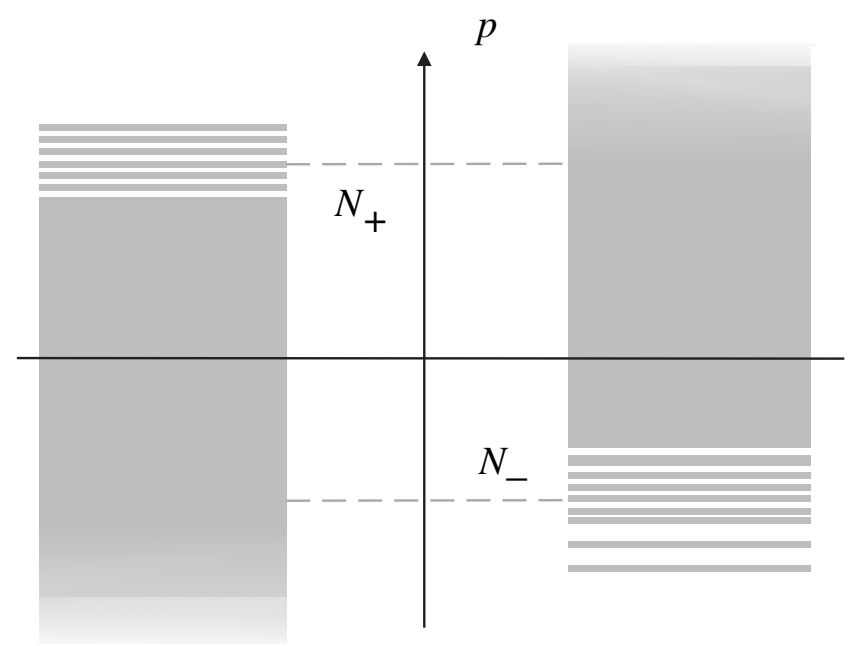

FIG. 2. In the large $N$ limit the two Fermi surfaces at $p=N_{+}$ and $p=-N_{-}$decouple and we obtain two independent relativistic free fermion field theories.

$$
N_{+}+N_{-}=N
$$

The partition function now simply factorizes, at least to all order in $1 / N$ as ${ }^{5}$

$$
Z_{N}\left(\theta, g_{s}\right)=\sum_{N_{+}+N_{-}=N} \psi_{N_{+}}\left(\theta, g_{s}\right) \cdot \bar{\psi}_{N_{-}}\left(\theta, g_{s}\right) .
$$

Here the chiral contribution $\psi_{N_{+}}$is captured by the zero fermion number sector of a two-dimensional chiral fermionic field theory. It is described by removing and adding arbitrary numbers of fermions close to the top Fermi surface. These states have momentum $p=N_{+}+k$ with $|k| \ll N$. Therefore their contribution to the partition function is given by

$$
g_{s} E=\frac{1}{2} g_{s} p^{2}=\mathrm{const}+N_{+} g_{s} k+\frac{1}{2} g_{s} k^{2} .
$$

Since the total number of particles and holes are equal, the constant contributions cancel. And in the large $N$ limit the quadratic term in $k$ is order $1 / N$ and can be ignored in the leading approximation. Therefore this sector can be described by a set of relativistic fermions with a linear dispersion relation. Including the $\theta$ term it is given by $t k$, with the complexified 't Hooft coupling

$$
t=g_{s} N_{+}+i \theta \text {. }
$$

The chiral partition function therefore becomes a holomorphic function of $t$

$$
\psi_{N_{+}}\left(\theta, g_{s}\right)=\psi\left(t, g_{s}\right)
$$

There is a similar term $\bar{\psi}_{N_{-}}\left(\theta, g_{s}\right)$ coming from the nega-

\footnotetext{
${ }^{5}$ There are nonperturbative contributions that preserve the factorized form, and they have been studied recently in $[40,41]$. In the next subsection, we will find other nonperturbative effects which break the factorized structure.
}

tive Fermi surface at $p=-N_{-}$. Here the moduli combine to give an antiholomorphic function $\bar{\psi}\left(\bar{t}, g_{s}\right)$ of

$$
\bar{t}=g_{s} N_{-}-i \theta .
$$

The quadratic corrections to the energy are controlled by $g_{s} \sim 1 / N$ and capture the perturbative string loop corrections.

\section{NONPERTURBATIVE CORRECTIONS TO CHIRAL FACTORIZATION}

The counting of states of two-dimensional Yang-Mills theory in terms of the chiral fermions is clearly only an approximation, because the two Fermi surfaces are not independent. Particles can move from the top to the bottom levels. These order $e^{-N}$ effects give a nonperturbative entanglement of the two chiral systems as we will now explain.

\section{A. Overcounting fermion configurations}

These corrections can be elegantly described as follows. In the large $N$ limit we are treating each of the two Fermi levels as the surface of an infinite deep sea, while in fact the sea is only of finite depth $N$. Therefore, we make mistakes if we create holes which lie too deep under the surface.

To be as concrete as possible, let us first decompose the partition function $Z_{N}$ in superselection sectors

$$
Z_{N}=\sum_{N_{+}+N_{-}=N} Z_{N_{+}, N_{-}},
$$

where $Z_{N_{+}, N_{-}}$receives contributions from configurations where there are $N_{+}$fermions with positive momenta $p_{i}>$ 0 and $N_{-}$fermions with negative momenta $p_{i}<0$. We can think of such a configuration as being created out of a Fermi sea, where the positive level is at $p=N_{+}$and the negative level at $p=-N_{-}$, as we did in the previous section. Now by convention we will assume that all the holes that are created with positive momentum have gone to excitations of the top Fermi surface, and that all the negative-momentum holes have gone to the bottom Fermi surface excitation.

If we denote the chiral amplitudes corresponding to this configuration as $\psi_{N_{+}}$and $\bar{\psi}_{N_{-}}$, then naïvely we can make the approximation

$$
Z_{N_{+}, N_{-}} \approx \psi_{N_{+}} \bar{\psi}_{N_{-}}
$$

However, this expression is clearly incomplete, since it will overcount states. Each chiral wave function does not limit the momenta of the holes to be positive for $\psi_{N_{+}}$or negative for $\bar{\psi}_{N_{-}}$, respectively. In $\psi_{N_{+}}$there are configurations where, for instance, $n_{-}$holes have been made with negative momenta in order to create $n_{-}$particles with positive momentum, raising the top level of Fermi sea from $N_{+}$to $N_{+}+n_{-}$. Similarly, $\bar{\psi}_{N_{-}}$takes into account states where $n_{+}$positive momentum holes are created that go to states 
with negative momentum, lowering the bottom level to $-N_{-}-n_{+}$. Typical examples of these "wrong" configurations are illustrated in Fig. 3(a).

In the Yang-Mills theory these configurations correspond to states with a total of $N_{+}^{\prime}=N_{+}+n_{-}-n_{+}$positive momentum fermions and a total of $N_{-}^{\prime}=N_{-}+n_{+}-n_{-}$negative-momentum fermions; see Fig. 3(b). These are perfectly fine states, but they have already been counted in $Z_{N_{+}^{\prime}, N_{-}^{\prime}}$, under the assumption that all positive momentum holes went to the top level and vice versa. Therefore, these states have to be subtracted from the product $\psi_{N_{+}} \bar{\psi}_{N_{-}}$.

Of course, Fig. 3(b) only depicts the corresponding ground state, that now has four Fermi surfaces. We note that the topology of the Fermi sea has changed. A "bubble" of holes is made with the Fermi sea. In general, each of these four Fermi surfaces will have fluctuations, as is illustrated in Fig. 4(a). In the large $N$ limit, and also when $n_{ \pm} \gg 1$, we can describe these fluctuations as essentially independent.

It is easy to derive in this way an exact recursion relation for the partition function $Z_{N}$ in terms of the mistakes made in the chiral approximation. As we have explained above, in that approximation we overcount a total number of $n=$ $n_{+}+n_{-}$holes that have been created on the "wrong side." Since a hole denotes the absence of a particle, their partition function is given by

$$
Z_{n}^{\text {holes }}\left(\theta, g_{s}\right)=Z_{n}\left(-\theta,-g_{s}\right)
$$

(We subsequently do not keep track of the $\theta$ dependence,

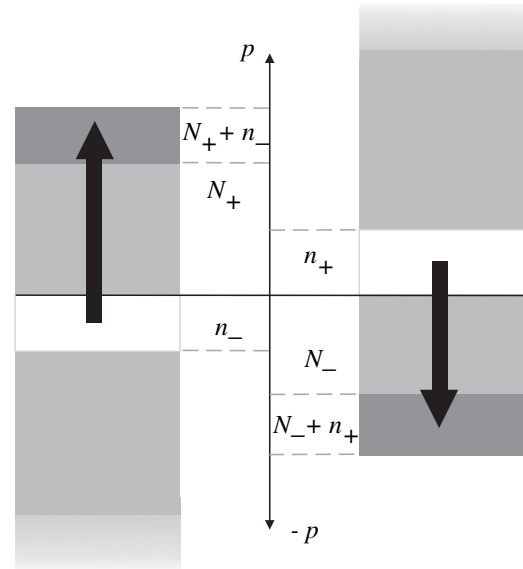

(a)

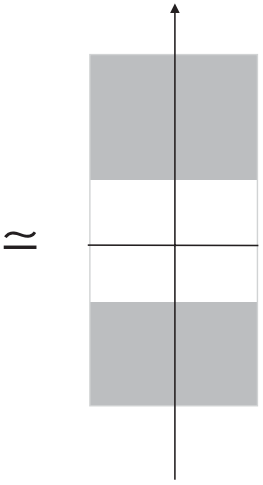

(b)
FIG. 3. The chiral product $\psi_{N_{+}} \bar{\psi}_{N_{-}}$overcounts states. As illustrated in (a), it contains configurations where $n_{-}$negativemomentum holes are brought to the top Fermi level and $n_{+}$ positive momentum holes are brought to the bottom level. These states, that look like (b) in the Yang-Mills theory, have already been counted and therefore have to be subtracted from the chiral product.

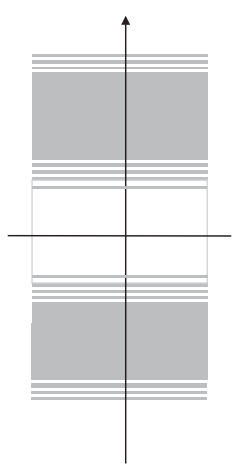

(a)

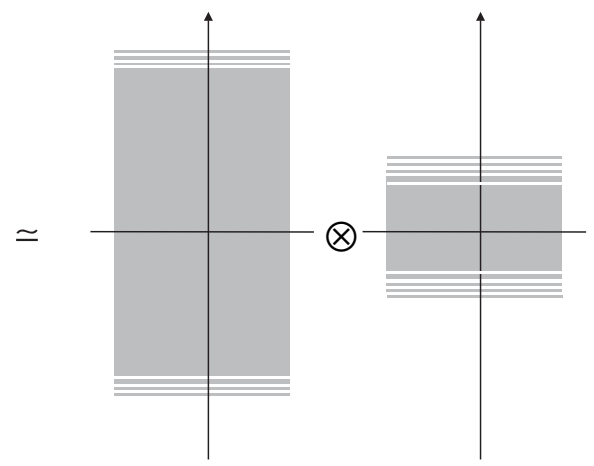

(b)
FIG. 4. The fundamental recursion relation: (a) The configurations that we have overcounted create a bubble of holes in the middle of the Fermi sea. (b) These states can be considered as a tensor product of two copies of the system, where the first factor consists of the usual particles, but the second factor is made out of holes.

which is identical for holes and particles by parity symmetry $\theta \rightarrow-\theta, p \rightarrow-p$.) On top of these holes we have the usual particle states. The total number of these particles is now $N+n$ and they are counted by the usual partition function $Z_{N+n}\left(g_{s}\right)$. Since the fermions are free, the total partition function of particles and holes is simply the tensor product. This is illustrated in Fig. 4(b). The partition function of these states comes with an extra minus sign, because the states are overcounted in the large $N$ chiral limit and therefore have to be subtracted. We thus derive the fundamental relation

$$
Z_{N}\left(g_{s}\right)=\sum_{k=0}^{N} \psi_{k}\left(g_{s}\right) \bar{\psi}_{N-k}\left(g_{s}\right)-\sum_{n>0} Z_{N+n}\left(g_{s}\right) Z_{n}\left(-g_{s}\right)
$$

Now as it stands, this formula really cannot be interpreted rigorously. Even though the left-hand side makes perfect sense for finite $N$, the chiral wave functions $\psi_{N}$ that appear on the right-hand side are only defined as an asymptotic expansion in $1 / N$. Therefore, the correction terms also must be considered as formal objects. This is clear, since we flipped the sign of $g_{s}=g_{\mathrm{YM}}^{2}$. This makes the theory ill-defined. ${ }^{6}$ We therefore have to give another, physically sensible interpretation of this result.

We will first sketch a rough argument and will then try to make more precise sense of this in the next section using formal generating functions. First of all, using CPT invariance, we can interpret the contributions of the holes as the partition function of a gauge theory of negative rank $U(-n)$ or equivalently as a supergroup of pure fermionic rank $0 \mid n$. That is, we can write

\footnotetext{
${ }^{6}$ This instability reminds one of Dyson's argument [42] about the nonanalyticity of the QED perturbative expansion: sending $e^{2} \rightarrow-e^{2}$ makes charged configurations unstable.
} 


$$
Z_{n}\left(-g_{s}\right)=Z_{-n}\left(g_{s}\right) .
$$

So the relation(3.1) can be written as

$$
Z_{N}\left(g_{s}\right)=\sum_{k=0}^{N} \psi_{k}\left(g_{s}\right) \bar{\psi}_{N-k}\left(g_{s}\right)-\sum_{n>0} Z_{N+n}\left(g_{s}\right) Z_{-n}\left(g_{s}\right) .
$$

Now we want to argue that, using a suitable analytic continuation, we can replace the sum over $n>0$ with a sum over $n<0$. In the next section will give an argument using formal generating functions. This last step $n \rightarrow-n$ will bring the relation to its final form

$$
Z_{N}\left(g_{s}\right)=\sum_{k=0}^{N} \psi_{k}\left(g_{s}\right) \bar{\psi}_{N-k}\left(g_{s}\right)-\sum_{n>0} Z_{N-n}\left(g_{s}\right) Z_{n}\left(g_{s}\right) .
$$

\section{B. Generating functions}

We will now give a more mathematical derivation of these results using generating functions.

\section{Warm-up: a case with linear dispersion relation}

Let us first consider a more elementary example to explain the main idea. Consider $N$ free fermions with a linear dispersion relation $E=p$. Here the momenta $p$ are half-integer and (to make the system stable) are taken to be positive, so $p \in \mathbf{Z}_{\geq 0}+\frac{1}{2}$. This system is related to the special case $m=0$ of the Calabi-Yau geometry (2.1). It is also exactly the spectrum of gauged matrix quantum mechanics with a quadratic potential [43], i.e., the $N \times N$ matrix harmonic oscillator with action

$$
S=\frac{1}{2} \int d t \operatorname{Tr}\left(\left(D_{t} \Phi\right)^{2}-\Phi^{2}\right) .
$$

The partition function is now defined as

$$
Z_{N}(t)=\sum_{\text {states }} e^{-t E}
$$

It is most simply written down in a grand canonical ensemble with chemical potential $\mu$. With $x=e^{-\mu}$ and $q=$ $e^{-t}$, it is given by the generating function

$$
Z(x ; t)=\sum_{N \geq 0} x^{N} Z_{N}(t)=\prod_{p>0}\left(1+x q^{p}\right) .
$$

In this case we have, of course, also a simple exact expression for $Z_{N}$ [43]

$$
Z_{N}(t)=q^{N^{2} / 2} \prod_{n=1}^{N}\left(1-q^{n}\right)^{-1} .
$$

When considered as a large $N$ string theory, there are only perturbative terms at genus zero and one. So, ignoring the nonperturbative effects, the perturbative answer in the large $N$ limit is given by (we suppress the dependence on $t$ in the following)

$$
\psi_{N}:=Z_{N}^{\text {pert }}=q^{N^{2} / 2} \prod_{n=1}^{\infty}\left(1-q^{n}\right)^{-1} .
$$

The notion of such a perturbative part only makes sense for large $N$. For example, to take an extreme case, $Z_{0}=1$, whereas according to the above definition $\psi_{0}$ would be given by the very nontrivial expression $\prod_{n>0}\left(1-q^{n}\right)^{-1}$.

It is easy to see that the nonperturbative corrections are of order $e^{-t N}=q^{N}$. One simply writes

$$
Z_{N}=\psi_{N} \cdot \prod_{n=1}^{\infty}\left(1-q^{N+n}\right) .
$$

These terms can indeed not be ignored for small $N$.

We will now derive a recursion relation for the exact partition function $Z_{N}$. Consider the Jacobi triple product identity (or boson-fermion correspondence)

$$
\prod_{n>0}\left(1-q^{n}\right) \prod_{p>0}\left(1+x q^{p}\right)\left(1+x^{-1} q^{p}\right)=\sum_{N \in \mathbf{Z}} q^{N^{2} / 2} x^{N} .
$$

With the above notation it can be written as

$$
Z(x) \cdot Z\left(x^{-1}\right)=\sum_{x \in \mathbf{Z}} x^{N} \psi_{N} .
$$

Let us also introduce a notation for the right-hand side, the generating function of the perturbative partition functions

$$
\psi(x)=\sum_{N \in \mathbf{Z}} x^{N} \psi_{N}
$$

Note that here $N$ is allowed to run also over negative integers, but $\psi_{-N}=\psi_{N}$. We repeat the caveat that there is a physical significance to $\psi_{N}$ only for large $N$.

With this notation we can now write the fundamental relation(3.4) even simpler as

$$
Z(x) \cdot Z\left(x^{-1}\right)=\psi(x) .
$$

Here $Z$ is the exact expression, $\psi$ the perturbative approximation; the relation is exact, though. When expanded back into components of fixed rank, it gives the recursion relation

$$
\sum_{k \geq 0} Z_{N+k} Z_{k}=\psi_{N}
$$

Since $Z_{0}=1$, we can write this more suggestively as

$$
Z_{N}=\psi_{N}-\sum_{k>0} Z_{N+k} Z_{k},
$$

where the second term on the right-hand side denotes the nonperturbative effects. Since $\psi_{n} \sim e^{-t n^{2} / 2}$, the $k$ th correction term in (3.6) is exponentially suppressed with respect to $\psi_{N}$ by a factor $e^{-t N k}$.

Now, if $N$ is large, the leading approximation is $Z_{N} \approx$ $\psi_{N}$. So, we can recursively expand the terms on the righthand side, at least if $k$ is also large, that is, of the order of $N$. 
Then we have

$$
Z_{N} \approx \psi_{N}-\sum_{k>0} \psi_{N+k} \psi_{k}+\mathcal{O}\left(\psi^{3}\right)
$$

Only the terms with $k \gg 1$ have a well-defined large $N$ expansion and can therefore be sensibly interpreted. This expansion of the recursion relation (3.6) continues. At the next order we have

$$
Z_{N} \approx \psi_{N}-\sum_{k>0} \psi_{N+k} \psi_{k}+\sum_{k_{1}, k_{2}>0} \psi_{N+k_{1}+k_{2}} \psi_{k_{1}} \psi_{k_{2}}+\mathcal{O}\left(\psi^{4}\right)
$$

\section{Two-dimensional Yang-Mills theory}

Now we consider the relevant case of two-dimensional Yang-Mills theory. Here the dispersion relation is quadratic $E=\frac{1}{2} p^{2}$ instead of linear, as in the previous case. The quadratic corrections give rise to the nontrivial string expansion.

Here too, it is most practical to consider the fermion system in a grand canonical ensemble with a chemical potential $\mu$ for the number of fermions $N$. Then we can write a compact generating function for the partition functions for all $U(N)$ theories at once. With the notation

$$
x=e^{-\mu}, \quad y=e^{i \theta}, \quad q=e^{-g_{s}},
$$

we then have an infinite product representation

$$
Z\left(x ; \theta, g_{s}\right)=\sum_{N \geq 0} x^{N} Z_{N}\left(\theta, g_{s}\right)=\prod_{p=-\infty}^{\infty}\left(1+x y^{p} q^{p^{2} / 2}\right) .
$$

Because $p$ runs over both positive and negative values, $Z$ is an even function of $\theta$.

In a similar way, there is a simple expression for the chiral partition function $\psi_{N_{+}}$that describes the fluctuation of the top Fermi surface at $p=N_{+} \gg 1$,

$$
\begin{aligned}
\psi_{N_{+}}\left(\theta, g_{s}\right)= & \oint \frac{d x}{2 \pi i x} x^{-N_{+}} \prod_{p>0}\left(1+x y^{p} q^{p^{2} / 2}\right) \\
& \times\left(1+x^{-1} y^{-p} q^{-p^{2} / 2}\right) .
\end{aligned}
$$

One can see easily from this product formula that the $N_{+}$ dependence of $\psi_{N_{+}}$is given by

$$
\begin{aligned}
\psi_{N_{+}}\left(\theta, g_{s}\right)= & y^{(1 / 2) N_{+}^{2}} q^{(1 / 6) N_{+}^{3}-(1 / 24) N_{+}} \\
& \cdot \psi_{0}\left(\theta-i g_{s} N_{+}, g_{s}\right) .
\end{aligned}
$$

The prefactor gives the leading energy and charge of the ground state. The second factor is the perturbative expansion and is only a function of $g_{s}$ and of $t=g_{s} N+i \theta$. So we can verify using these explicit formulas that, as claimed,

$$
\psi_{N_{+}}\left(\theta, g_{s}\right)=\psi\left(t, g_{s}\right)
$$

with

$$
\begin{aligned}
\psi\left(t, g_{s}\right)= & \oint \frac{d x}{2 \pi i x} \prod_{p>0}\left(1+x e^{-t p} q^{p^{2} / 2}\right) \\
& \times\left(1+x^{-1} e^{-t p} q^{-p^{2} / 2}\right) .
\end{aligned}
$$

For the bottom Fermi surface at $p=-N_{-}$we have similarly (with $\bar{t}=g_{s} N_{-}-i \theta$ )

$$
\begin{aligned}
\bar{\psi}\left(\bar{t}, g_{s}\right):= & \bar{\psi}_{N_{-}}\left(\theta, g_{s}\right) \\
= & \oint \frac{d x}{2 \pi i x} \prod_{p>0}\left(1+x e^{-\bar{t} p} q^{p^{2} / 2}\right) \\
& \times\left(1+x^{-1} e^{-\bar{t} p} q^{-p^{2} / 2}\right) .
\end{aligned}
$$

Again, these expressions only make sense at large $N$, as an expansion in $1 / N$.

We will now repeat some of the previous steps that we performed in the linear case. Consider the formal product

$$
\begin{aligned}
Z\left(x ; \theta, g_{s}\right) \cdot Z\left(x^{-1} ;-\theta,-g_{s}\right)= & \prod_{p=-\infty}^{+\infty}\left(1+x y^{p} q^{p^{2} / 2}\right) \\
& \times\left(1+x^{-1} y^{-p} q^{-p^{2} / 2}\right) .
\end{aligned}
$$

Here the second factor can be considered as a partition function for holes. By CPT it is obtained by reversing the signs of all potentials

$$
\left(\mu, \theta, g_{s}\right) \rightarrow\left(-\mu,-\theta,-g_{s}\right) .
$$

This hole factor is clearly problematic when viewed as a power series in $q$, since the holes can have arbitrary negative energy $-\frac{1}{2} p^{2}$. It diverges badly. At this point we can therefore only consider it as a formal expansion in powers of $g_{s}$.

Now consider the Laurent expansion in $x$ of the above product. This is best done by splitting the product over all $p$ in (3.10) in a product over $p>0$ and a product over $p<0$. Then, comparing to the expression (3.8) for the chiral wave function, we see that (suppressing the $\theta$ dependence in our notation)

$$
Z\left(x ; g_{s}\right) Z\left(x^{-1} ;-g_{s}\right)=\sum_{N_{+}, N_{-} \geq 0} x^{N_{+}+N_{-}} \psi_{N_{+}}\left(g_{s}\right) \bar{\psi}_{N_{-}}\left(g_{s}\right) .
$$

Now we can formally expand the contribution of the holes as

$$
Z\left(x^{-1} ;-g_{s}\right)=\sum_{N \geq 0} x^{-N} Z_{N}\left(-g_{s}\right) .
$$

This gives an identity of the form

$$
\sum_{n \geq 0} Z_{N+n}\left(g_{s}\right) Z_{n}\left(-g_{s}\right)=\sum_{k=0}^{N} \psi_{k}\left(g_{s}\right) \bar{\psi}_{N-k}\left(g_{s}\right) .
$$


Using $Z_{0}=1$ we thus recover relation (3.5)

$$
Z_{N}\left(g_{s}\right)=\sum_{k=0}^{N} \psi_{k}\left(g_{s}\right) \bar{\psi}_{N-k}\left(g_{s}\right)-\sum_{n \geq 1} Z_{N+n}\left(g_{s}\right) Z_{n}\left(-g_{s}\right) .
$$

Now there is a way to make more sense of the holes factor $Z\left(x ;-\theta,-g_{s}\right)$. Formally, using the identity

$$
\left(1+a^{-1}\right)=a^{-1}(1+a),
$$

we can write

$$
\begin{aligned}
Z\left(x^{-1} ;-\theta,-g_{s}\right)= & \prod_{p=-\infty}^{\infty}\left(1+x^{-1} y^{p} q^{-p^{2} / 2}\right) \\
= & x^{a} y^{b} q^{c} \prod_{p=-\infty}^{\infty}\left(1+x y^{-p} q^{p^{2} / 2}\right) \\
& \sim Z\left(x ; \theta, g_{s}\right) .
\end{aligned}
$$

Here the $a, b, c$ are some constants, strictly speaking all infinite. But we could assume that they are given by, for example, zeta-function regularization. Since the sum runs over both positive and negative $p$ these regulated sums are actually zero.

In fact, we will turn things around and define $Z\left(-g_{s}\right)$ through this procedure. Note that Eq. (3.11) now becomes

$$
Z\left(x ; \theta, g_{s}\right)^{2}=\sum_{N_{+}, N_{-} \geq 0} x^{N_{+}+N_{-}} \psi_{N_{+}}\left(g_{s}\right) \bar{\psi}_{N_{-}}\left(g_{s}\right)
$$

or, when written in components, reproduces (3.3):

$$
Z_{N}\left(g_{s}\right)=\sum_{k=0}^{N} \psi_{k}\left(g_{s}\right) \bar{\psi}_{N-k}\left(g_{s}\right)-\sum_{n>0} Z_{N-n}\left(g_{s}\right) Z_{n}\left(g_{s}\right) .
$$

As in the previous example, we can only trust this formula in the large $N$ limit and for the terms with $k \gg 1$ finite.

We can iteratively solve (3.13) for $Z_{N}$ in a power series expansion in $\psi_{k} \bar{\psi}_{N-k}$ as

$$
\begin{aligned}
Z_{N}= & \sum_{n=1}^{\infty}(-1)^{n-1} C_{n-1} \sum_{N_{+}^{1}+\cdots+N_{+}^{n}+N_{-}^{1}+\cdots+N_{-}^{n}=N} \\
& \times \psi_{N_{+}^{1}} \cdots \psi_{N_{+}^{n}} \bar{\psi}_{N_{-}^{1}} \cdots \bar{\psi}_{N_{-}^{n}},
\end{aligned}
$$

where $C_{n}$ in the coefficient is the Catalan number,

$$
C_{n}=\frac{(2 n) !}{n !(n+1) !} .
$$

This combinatorial factor arises since the generating function $C(x)=\sum_{n=1}^{\infty} C_{n-1} x^{n}$ for the Catalan number obeys the quadratic equation,

$$
C(x)=x+C(x)^{2},
$$

which has the same structure as (3.13) with the identification $x \rightarrow-\psi \bar{\psi}$ and $C \rightarrow-Z$. The Calatan number $C_{n-1}$ is known to be equal to the number of binary bracketings of $n$ letters. Or, put differently, it counts the number of ways to create a planar binary tree with $n$ branches. An interpretation of this combinatorial coefficient in the context of the gravity dual will be discussed in Sec. V.

Note that the expansion (3.14) is reliable only in the regime where the baby universe number $k$ is much larger than the corresponding parent $K$ and much bigger than one, $K \gg k \gg 1$. This gives a hierarchical structure to the various terms in (3.14), which will be important for a gravity interpretation of the coefficients $C_{n-1}$.

\section{A convenient rewriting of the result}

In this section we recast the above result in a convenient form which will be more immediately applicable to our holographic gravitational interpretation.

Let us recall that the number of BPS degeneracies of D4, D2-, and D0-branes $\Omega\left(N_{4}, N_{2}, N_{0}\right)$ can be computed by Fourier transform of the Yang-Mills answer. In particular (with $N_{4}=N$ )

$$
\begin{aligned}
\Omega\left(N, N_{2}, N_{0}\right)= & \int d\left(\frac{1}{g_{s}}\right) d\left(\frac{\theta}{g_{s}}\right) \\
& \times \exp \left(\frac{4 \pi^{2} N_{0}}{g_{s}}+\frac{2 \pi N_{2} \theta}{g_{s}}\right) Z_{N}\left(g_{s}, \theta\right) .
\end{aligned}
$$

Now, let us define

$$
\begin{aligned}
\psi_{N, N_{2}, N_{0}}\left(\theta, g_{s}\right)= & \exp \left(\frac{2 \pi^{2} N_{0}}{g_{s}}+\frac{N_{2} \pi \theta}{g_{s}}\right) \\
& \times \psi\left(\frac{1}{2} N g_{s}+i \theta, g_{s}\right),
\end{aligned}
$$

which we sometimes also denote as

$$
\psi_{N, N_{2}, N_{0}}\left(\theta, g_{s}\right)=\left\langle N, N_{2}, N_{0}, \theta, g_{s} \mid \psi\right\rangle .
$$

This is the wave function of the topological string in the corresponding flux sector. For brevity of notation, sometimes we use the following notation,

$$
\left|N, N_{2}, N_{0}\right\rangle=\left\langle N, N_{2}, N_{0} \mid \psi\right\rangle .
$$

In other words in this notation we would have

$$
\psi_{N, N_{2}, N_{0}}\left(\theta, g_{s}\right)=\left\langle\theta, g_{s} \mid N, N_{2}, N_{0}\right\rangle,
$$

which we hope does not cause confusion.

Then keeping the leading all order in the $1 / N$ expansion we can write (3.17) as

$$
\Omega\left(N, N_{2}, N_{0}\right)=\int d\left(\frac{1}{g_{s}}\right) d\left(\frac{\theta}{g_{s}}\right)\left|\psi_{N, N_{2}, N_{0}}\left(\theta, g_{s}\right)\right|^{2} .
$$

In other words, to all orders in the $1 / N$ expansion

$$
\Omega\left(N, N_{2}, N_{0}\right)=\left\langle N, N_{2}, N_{0} \mid N, N_{2}, N_{0}\right\rangle .
$$

Note that the extra sum over $k$ in shifting $N$ up and down between $\psi$ and $\bar{\psi}$ is already incorporated by the integral 
over $\theta / g_{s}$ (which is best seen by analytic continuation), and does not need to be included on top of this.

Now we come to using the formula (3.13) to incorporate effects which are of order $\exp (-t N)$ and smaller. $^{7}$ However the corrections involve more than one copy of $\psi$ and $\bar{\psi}$. This suggests that we try to interpret this as multiHilbert space corrections.

For simplicity let us start with the first application of the recursion formula to (3.13). The recursion relation leads at this order to (where we suppress the extra shifting of $N$ between $\psi$ and $\bar{\psi}$ as that would be automatically taken care of by the inverse Fourier transform discussed above)

$$
-\left|\psi_{N-k}\left(\theta, g_{s}\right)\right|^{2}\left|\psi_{k}\left(\theta, g_{s}\right)\right|^{2} .
$$

In order to give this expression a Hilbert space interpretation we introduce additional variables which are gotten rid of by additional delta function integrals:

$$
\begin{aligned}
\Omega\left(N, N_{2}, N_{0}\right)_{2}= & -\sum_{k>0} \int d\left(\frac{\theta}{g_{s}}\right) d\left(\frac{1}{g_{s}}\right) d\left(\frac{\theta^{\prime}}{g_{s}^{\prime}}\right) d\left(\frac{1}{g_{s}^{\prime}}\right) \\
& \times \delta\left(\frac{\theta^{\prime}}{g_{s}^{\prime}}-\frac{\theta}{g_{s}}\right) \delta\left(\frac{1}{g_{s}^{\prime}}-\frac{1}{g_{s}}\right)\left|\psi_{N-k}\left(\theta, g_{s}\right)\right|^{2} \\
& \times\left|\psi_{k}\left(\theta^{\prime}, g_{s}^{\prime}\right)\right|^{2} \exp \left(+N_{2} \theta / g_{s}+N_{0} / g_{s}\right) .
\end{aligned}
$$

Next we write each of the delta functions (taking the periodicities of the chemical potential into account) as $\delta(X)=\sum_{m} e^{m X}$, which leads to rewriting the above as

$$
\begin{aligned}
\Omega\left(N, N_{2}, N_{0}\right)_{2}= & -\sum_{k>0, m, p} \int d\left(\theta, g_{s}, \theta^{\prime}, g_{s}^{\prime}\right) \\
& \times\left|\psi_{N-k, N_{2}-m, N_{0}-p}\left(\theta, g_{s}\right)\right|^{2} \\
& \times\left|\psi_{k, m, p}\left(\theta^{\prime}, g_{s}^{\prime}\right)\right|^{2} .
\end{aligned}
$$

This result can be summarized in the notation we introduced before as

$$
\begin{gathered}
\Omega\left(N, N_{2}, N_{0}\right)_{2}=-\sum_{k>0, m, p}\left\langle N-k, N_{2}-m, N_{0}-p\right| N \\
\left.-k, N_{2}-m, N_{0}-p\right\rangle \\
\cdot\langle k, m, p \mid k, m, p\rangle .
\end{gathered}
$$

The generalization to arbitrary orders in the recursive relation is also clear. We define a state in the sum of arbitrary number of copies of the Hilbert space. We define

$$
\left|\psi_{n}\right\rangle=\sum_{N_{4}^{i}, N_{2}^{i}, N_{0}^{i}} \bigotimes_{i=1}^{n}\left|N_{4}^{i}, N_{2}^{i}, N_{0}^{i}\right\rangle
$$

where each term in the sum is restricted by the condition

\footnotetext{
${ }^{7}$ Note that this also explains why the four-dimensional black hole interpretation of topological strings is not a good starting point for degeneracies of five-dimensional black holes where one may take a small number of magnetic branes $N \sim 1$, because these corrections are of order 1.
}

that

$$
\sum_{i} N_{4}^{i}=N, \quad \sum_{i} N_{2}^{i}=N_{2}, \quad \sum_{i} N_{0}^{i}=N_{0},
$$

and where all $N_{4}^{i}>0$. Our result is then summarized as

$$
\Omega\left(N, N_{2}, N_{0}\right)=\sum_{n}(-1)^{n-1} C_{n-1}\left\langle\psi_{n} \mid \psi_{n}\right\rangle .
$$

We will give an interpretation of this result in Sec. V, after we discuss some aspects of the holographically dual gravity solutions.

\section{GRAVITY INTERPRETATION}

In this section we will consider a type IIB compactification on a Calabi-Yau manifold $M$. Mirror symmetry will relate this to an equivalent description within type IIA, which we can use to compare with the gauge theory result in the last section.

Let us start by describing the standard single center black hole solution at the string tree level. Consider a static spherically symmetric metric,

$$
d s^{2}=-\frac{\pi}{S(r)} d t^{2}+\frac{S(r)}{\pi} \sum_{a=1,2,3}\left(d x^{a}\right)^{2}+d s_{\mathrm{CY}}^{2},
$$

where $r=|x|$. The metric $d s_{\mathrm{CY}}^{2}$ of the Calabi-Yau threefold also can depend on $r$, so we have a "warped compactification." Let $X^{I}\left(I=0,1, \ldots, h^{2,1}\right)$ be the projective coordinates of the complex structure moduli space of the Calabi-Yau manifold normalized as

$$
S=-\frac{\pi}{2} \operatorname{Im} \tau_{I J} X^{I} \bar{X}^{J}=\frac{\pi}{2} \operatorname{Im}\left[X^{I} \bar{\partial}_{I} \bar{F}_{0}\right],
$$

where $F_{0}(X)$ is the genus 0 topological string partition function and $\tau_{I J}=\partial_{I} \partial_{J} F_{0}$ is the period matrix of the Calabi-Yau three-fold. Assuming that $X^{I}$ are also functions of $r$, the classical BPS black hole solution with electric charge $Q_{I}$ and magnetic charge $P^{I}$ is given by

$$
\operatorname{Re} X^{I}=\frac{P^{I}}{|x|}+c^{I}, \quad \operatorname{Re}_{I} F_{0}=\frac{Q_{I}}{|x|}+d_{I},
$$

where $c^{I}, d_{I}$ are integration constants that correspond to values of the Calabi-Yau moduli at spatial infinity. This shows how the Calabi-Yau metric $d s_{\mathrm{CY}}^{2}$ depends on $r=$ $|x|$. (The Kähler moduli are kept constant.) The horizon is at $x \rightarrow 0$, where $X^{I}$ approaches the attractor point [44]:

$$
\operatorname{Re} X^{I} \sim \frac{P^{I}}{|x|}, \quad \quad \operatorname{Re} \partial_{I} F_{0} \sim \frac{Q_{I}}{|x|},
$$

independently of their values at the infinity. The semiclassical entropy for this solution is given by

$$
\begin{aligned}
S_{\mathrm{BH}}^{(0)}(P, Q) & =\frac{\pi}{2} \operatorname{Im}\left[X^{I} \bar{\partial}_{I} \bar{F}_{0}\right] \\
& =F_{0}(X)+\bar{F}_{0}(\bar{X})+\pi \sum_{I} Q_{I} \operatorname{Im} X^{I},
\end{aligned}
$$


where $X^{I}$ 's are fixed by (4.4). This is also related to the asymptotic behavior of $S(x)$,

$$
S(x) \sim \frac{S_{\mathrm{BH}}^{(0)}(P, Q)}{|x|^{2}}, \quad x \rightarrow 0,
$$

and for this reason we may regard $S(x)$ as a "position dependent entropy." The near-horizon geometry is $\mathrm{AdS}_{2} \times$ $S^{2} \times M$, where the complex structure moduli of the CalabiYau three-fold $M$ are fixed by the attractor Eqs. (4.4).

String loop effects modify the low energy effective action, and the black hole geometry is also changed accordingly. In [7], it was shown how to systematically take these perturbative effects into account. Remarkably the entropy formula to all order in the perturbative expansion can be concisely expressed in terms of the topological string partition function $F_{\text {top }}(X)$,

$$
F_{\text {top }}(X)=\sum_{g=0}^{\infty} F_{g}(X)
$$

where $F_{g}$ is the genus $g$ partition function. In [8] it was shown that the string loop corrected entropy $S_{\mathrm{BH}}(P, Q)$ of [7] can be expressed simply as

$$
S_{\mathrm{BH}}(P, Q)=F_{\text {top }}(X)+\bar{F}_{\text {top }}(\bar{X})+\sum_{I} Q_{I} \Phi^{I},
$$

where the Calabi-Yau moduli are fixed to be

$$
X^{I}=P^{I}+\frac{i}{\pi} \Phi^{I},
$$

and $\Phi^{I}$ 's are nonlinearly related to the charges $(P, Q)$ by

$$
Q_{I}=-\frac{\partial}{\partial \Phi^{I}}\left[F_{\text {top }}(X)+\bar{F}_{\text {top }}(\bar{X})\right] .
$$

This generalizes the classical entropy formula (4.5), and a quantum version of the attractor equation is given by (4.8) and (4.9). One can regard (4.7) as the Legendre transformation between the entropy, which depends on $P$ and $Q$, and the topological string partition function $F_{\text {top }}+\bar{F}_{\text {top }}$, which naturally is a function of $P$ and $\Phi$. Motivated by this observation, and the earlier work [7], it was conjectured in[8] that the number $\Omega(P, Q)$ of microscopic BPS states of the black hole is given by the Laplace transformation of the topological string partition function as

$$
Z_{\mathrm{BH}} \equiv \sum_{q} \Omega(P, Q) e^{-Q_{I} \Phi^{I}}=\left|\psi_{\text {top }}(X)\right|^{2},
$$

where

$$
\psi_{\text {top }}(X)=\exp F_{\text {top }}(X)
$$

and $X^{I}$ 's are given by (4.8).

The main purpose of this paper is to understand nonperturbative corrections to this formula. In this section, we will discuss sources of such corrections from the gravity point of view, and compare them with the results we found in the gauge theory point of view in the last section.

\section{A. Multicenter solutions}

The crucial observation is that the spherically symmetric geometry given by (4.1), (4.2), and (4.3) is not the only solution for a given set of charges $(P, Q)$ that preserves half of the supersymmetry. In fact there are multicenter solutions satisfying the same asymptotic behavior at spatial infinity [22]. Each of these solutions is characterized by a decomposition of the charges $(P, Q)$ as

$$
P^{I}=\sum_{i=1}^{n} P_{i}^{I}, \quad Q_{I}=\sum_{i=1}^{n} Q_{i I}
$$

where $n$ is the number of disjoint horizons of the solution and $\left(P_{i}, Q_{i}\right)$ are charges associated to each horizon $(i=$ $1, \ldots, n)$.

Before describing general multicenter solutions, it would be instructive to discuss the simplest case when there is only one gauge field and no scalar field. (This corresponds to the type IIB compactification on a rigid Calabi-Yau manifold, $h^{2,1}=0$.) Furthermore, suppose that the black hole carries no magnetic charge, i.e. $P=0$. Multicenter solutions in this case have been known for a long time, and they are called conformastatic solutions [45]. When there are several extremal black holes whose charges are of equal sign, their gravitational attraction is balanced by the Coulomb repulsion, and they can be placed at arbitrary positions in three spatial dimensions and still remain static. Such a solution can be constructed as follows. Consider a scalar function $S(x)$ of the form

$$
S(x)=\pi\left(c+\sum_{i=1}^{n} \frac{Q_{i}}{\left|x-x_{i}\right|}\right)^{2},
$$

whose square-root solves the Laplace equation $\Delta \sqrt{S(x)}=$ 0 in three dimensions. We choose $Q_{i}$ to be all positive and the constant $c$ is also positive, so that $S(x)$ never vanishes. The metric is then given by

$$
d s^{2}=-\frac{\pi}{S(x)} d t^{2}+\frac{S(x)}{\pi} \sum_{a=1,2,3}\left(d x^{a}\right)^{2}+d s_{\mathrm{CY}}^{2}
$$

The function $\sqrt{S(x)}$ also serves as the scalar potential for the gauge field. This describes a collection of extremal black holes with charge $Q_{i}$ at $x_{i}$. In fact, as $x$ approaches $x_{i}$, $S(x)$ behaves as

$$
S(x) \sim \frac{\pi Q_{i}^{2}}{\left|x-x_{i}\right|^{2}},
$$

where the geometry approaches that of $\mathrm{AdS}_{2} \times S^{2}$ with charge $Q_{i}$. Toward spatial infinity, we have

$$
S(x) \sim \pi\left(c+\frac{Q}{|x|}+\cdots\right)^{2}, \quad|x| \rightarrow \infty,
$$


where

$$
Q=\sum_{i=1}^{n} Q_{i}
$$

and therefore the solution has the same asymptotic behavior as the single center solution with the charge $Q$. These multicenter solutions also preserve one half of the supersymmetry.

It was pointed out in [46] that, in the limit of $c \rightarrow 0$, Wick rotation of the solution given by (4.13) and (4.14) describes quantum tunneling of a single universe into several disjoint universes. After the Wick rotation,

$$
d s_{E}^{2}=\frac{\pi}{S(x)} d \tau^{2}+\frac{S(x)}{\pi} \sum_{a=1,2,3}\left(d x^{a}\right)^{2}+d s_{\mathrm{CY}}^{2},
$$

it is convenient to think of $S(x)$ as the Euclidean time. For $S \rightarrow 0$, the geometry approaches to $\mathrm{AdS}_{2} \times S^{2}$ with charge $Q$. On the other hand, as $S \rightarrow \infty$, the geometry fragmented into pieces located at $x_{i}(i=1, \ldots, n)$, each of which has the form of $\mathrm{AdS}_{2} \times S^{2}$ with charges $Q_{i}$. The Euclidean action $I$ of this solution was evaluated in [46] as

$$
I=\pi \sum_{i<j} Q_{i} Q_{j}
$$

It was noted in there that this can be written as

$$
I=-\frac{1}{2}\left(S_{\mathrm{BH}}^{(0)}\left(Q_{1}\right)+\cdots+S_{\mathrm{BH}}^{(0)}\left(Q_{n}\right)-S_{\mathrm{BH}}^{(0)}(Q)\right),
$$

where $S_{\mathrm{BH}}^{(0)}$ is the semiclassical entropy given by (4.5). This suggests $[24,46]$ that $e^{-I}$ is an instanton amplitude for a tunneling of a single universe with charge $Q$ into $n$ universes with charges $Q_{1}, \ldots, Q_{n}$ so that its square gives the transition probability expected from the detailed balance argument. We will find that the comparison with the gauge theory computation in the previous section supports this interpretation.

This construction can be generalized to the case with $h^{2,1} \geq 1$ as follows. There are $\left(h^{2,1}+1\right)$ gauge fields and $h^{2,1}$ scalar fields describing the complex structure of the Calabi-Yau manifold. First consider the case when the charge vectors $\left(P_{i}, Q_{i}\right)$ are all parallel to each other. In this case, we are in practice turning on only one linear combination of the gauge fields. Since the attractor fixed point depends only on ratios of charges, the complex structure of the Calabi-Yau are kept constant. In this case, the horizons can still be located at arbitrary points.

The situation is more subtle with nonparallel charges $[22,47]$. In this case, Calabi-Yau moduli at each horizon can be different, and it costs kinetic energy for the scalar fields to interpolate between the different horizon values. Moreover the electromagnetic interaction does not completely balance the gravitational attraction. The metric in this case takes the form

$$
d s^{2}=-\frac{\pi}{S(x)}\left(d t+\omega_{i} d x^{i}\right)^{2}+\frac{S(x)}{\pi} \sum_{i=1,2,3}\left(d x^{i}\right)^{2}+d s_{\mathrm{CY}}^{2} .
$$

As before, the function $S(x)$ and the Calabi-Yau moduli are combined into $\left(h^{2,1}+1\right)$ variables $X^{I}(x)$ normalized as in (4.2). Defining $Q_{I}(x)$ and $P^{I}(x)$ by

$$
P^{I}(x)=\sum_{i=1}^{n} \frac{P_{i}^{I}}{\left|x-x_{i}\right|}+c^{I}, \quad Q_{I}(x)=\sum_{i=1}^{n} \frac{Q_{i I}}{\left|x-x_{i}\right|}+d_{I},
$$

$X^{I}$ are determined by

$$
\operatorname{Re} X^{I}=P^{I}(x), \quad \operatorname{Re}_{I} F_{0}=Q_{I}(x) .
$$

Thus the Calabi-Yau metric $d s_{\mathrm{CY}}^{2}$ also depends on $x$. The function $S(x)$ is determined by

$$
S(x)=\frac{\pi}{2} \operatorname{Im}\left[X^{I} \bar{\partial}_{I} \bar{F}_{0}\right] .
$$

The off-diagonal component in the metric is found by solving

$$
* d \omega=\sum_{I}\left(P^{I}(x) d Q_{I}(x)-Q_{I} d P^{I}(x)\right),
$$

where $*$ is the Hodge star operator with respect to the flat metric on $\mathbf{R}^{3}$. The integrability of (4.21) leads to a set of constraints on the locations of the horizons:

$$
\sum_{j \neq i} \frac{e_{i j}}{\left|x_{i}-x_{j}\right|}=\sum_{I} \operatorname{Re}\left(Q_{i I} c^{I}-P_{i}^{I} d_{I}\right), \quad i=1, \ldots, n,
$$

where

$$
e_{i j}=\sum_{I}\left(Q_{i I} P_{j}^{I}-P_{i}^{I} Q_{j I}\right)
$$

It should be noted that, in addition to this constraint, we need to require that the factor $S(x)$ in the metric remains nonzero. With (4.2), (4.19), and (4.20), requiring this for all $x \in \mathbf{R}^{3}$ implies an inequality on the charges $\left(P_{i}^{I}, Q_{i I}\right)$. We will see that, in the example we studied in the last section, this constraint agrees with what we found in the gauge theory side.

As in the single charge case discussed at (4.15), the Wick rotation $-d t^{2} \rightarrow+d \tau^{2}$ of(4.18) gives a metric which is asymptotically flat at spatial infinity and fragments into several $\mathrm{AdS}_{2}$ throats for $S \rightarrow \infty$ (see Fig. 5). Because of the off-diagonal term $\omega_{i} d x^{i} d t$, the metric in general becomes complex-valued after the Wick rotation. Since it becomes real in the asymptotic regions, it is still appropriate to use it as a saddle point and it can contribute to the functional integral [48]. 


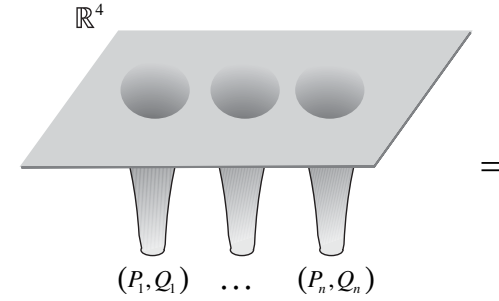

(a)

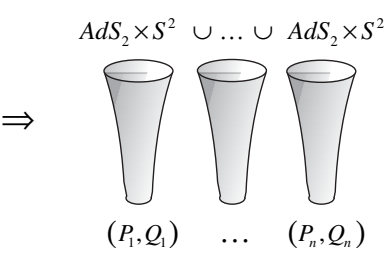

(b)
FIG. 5. The metric (4.18) is sketched in (a), it describes an asymptotically flat space-time with multiple black holes with charges $\left(P_{i}^{I}, Q_{i I}\right)(i=1, \ldots, n)$. In the near-horizon limit (b) this geometry gives a disjoint sum of $n \mathrm{AdS}_{2} \times S^{2}$ geometries.

\section{B. Baby universes interpretation}

How should we interpret the existence of such multicenter solutions? It is reasonable to expect that the Euclidean functional integral contains a sum over these solutions in the saddle point approximation. In the standard AdS/CFT correspondence, the low energy limit on Dbranes is dual to the near-horizon limit in the gravity side. For the multicenter metric, a natural near-horizon limit gives $n$ disjoint universes, each with the $\mathrm{AdS}_{2} \times S^{2} \times$ $M$ geometry, and the moduli of the Calabi-Yau three-fold $M$ at each universe are fixed by the attractor equations. Thus, we are led to conjecture that the full partition function of the gravity theory contains a sum over disjoint universes (baby universes); see Fig. 5.

Note that if we consider the projection of our geometry to the $S$-line, and consider the Euclidean space which is the pre-image of the Euclidean time $S$, we obtain the topology of a branched tree, where $S$ can be viewed as the "height" of the tree, as shown in Fig. 6. The number of components of the space for a given value of $S$ denotes the number of branches of the tree at a fixed height. As $S \rightarrow \infty$ the number of branches equals $n$, the number of black hole centers, see Fig. 7.

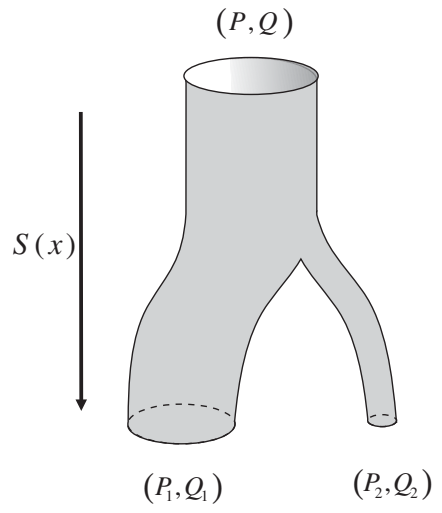

FIG. 6. As a function of Euclidean time, which can be identified with the local entropy $S(x)$ of the metric (4.18), the geometry describes the branching off of baby universes.

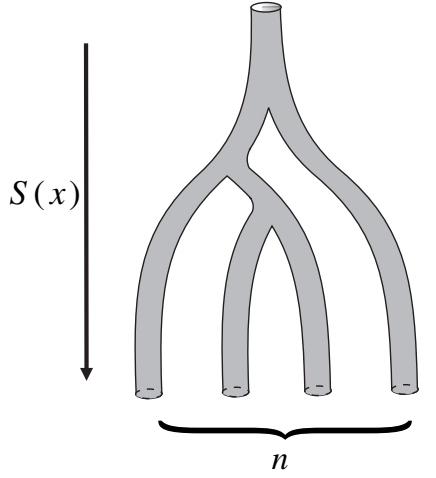

FIG. 7. As $S \rightarrow \infty$, the number of branches increases to $n$, the number of black hole centers.

In fact with a fixed asymptotic flux, we are forced to do this summation since there are instantons which change the number of baby universes [24,46] as we saw earlier. Since each $\mathrm{AdS}_{2}$ throat contributes to the partition function by a factor of $\exp S_{\mathrm{BH}}\left(Q_{i}, P_{i}\right)(i=1, \ldots, n)$, the total partition function should be of the form,

$$
\begin{aligned}
Z_{\mathrm{BH}}= & \sum_{n=1}^{\infty} C_{n-1} \sum_{\substack{P=P_{1}+\cdots+P_{n} \\
Q=Q_{1}+\cdots+Q_{n}}} \exp \left(S_{\mathrm{BH}}\left(Q_{1}, P_{1}\right)+\cdots\right. \\
& \left.+S_{\mathrm{BH}}\left(Q_{n}, P_{n}\right)\right) .
\end{aligned}
$$

The sum over charges may be restricted by the condition that the gravity solution exists. The coefficient $C_{n-1}$ is the Catalan number and reflects the distinct planar trees (where on each node of the tree the parent universe splits off say to the left) with $n$ branches which lead to this baby universe sum. This would be a valid description at least in the regime where we have a hierarchical splitting of parent universes to baby universes, i.e. when the charges of the parent universe are much larger than those of the baby universes as discussed at the end of Sec. III B.

We expect a holographic dual to the gravity theory to capture this branching process, and we should be able to interpret the different contributions in the gauge theory as coming from the different geometries that can contribute to the Euclidean functional integral. Moreover, since the latter have finite action, this translates into gauge theory configurations which are weighted by $e^{-N}$ with respect to the vacuum configuration. In the next section, we will show that the $e^{-N}$ terms in the gauge theory discussed in the previous section indeed correspond to baby universes and have the right hierarchical branching structure of (4.24). Interestingly, we will find that the gauge theory predicts an additional sign factor of $(-1)^{n-1}$ in (4.24).

\section{COMPARISON BETWEEN GAUGE THEORY AND GRAVITY THEORY}

In the previous section we have argued that the D-brane gauge theory is holographically dual not to a single black 
hole, but to an arbitrary ensemble of extremal black holes with a given total flux. In other words, fixing the flux at infinity allows more than a single black hole solution. This was used to suggest that the gauge theory is holographically dual not to a single $\mathrm{AdS}_{2} \times S^{2}$ but to an ensemble of $\mathrm{AdS}_{2} \times S^{2}$ 's. For each horizon the arguments of [8] apply and

$$
\exp S(P, Q)=\langle P, Q \mid P, Q\rangle
$$

where

$$
\langle\Phi \mid P, Q\rangle=e^{-(1 / 2) Q \cdot \Phi} \psi_{\mathrm{top}}\left(P+\frac{i}{\pi} \Phi\right)=\psi_{P, Q}(\Phi) .
$$

Using this we can rewrite the relation (4.24) as

$$
\Omega(P, Q)=\sum_{n=1}^{\infty} C_{n-1} \sum_{P_{i}, Q_{i}} \bigotimes_{i=1}^{n}\left\langle P_{i}, Q_{i} \mid P_{i}, Q_{i}\right\rangle
$$

where $\sum P_{i}=P$ and $\sum Q_{i}=Q$ and with the restriction that the gravity solutions should exist. This prediction also can be stated using the picture proposed in [12] for the Hartle-Hawking wave function in the mini-superspace. The case considered there involved type IIB string theory on nine-dimensional space being

$$
X=S^{1} \times S^{2} \times M,
$$

where $M$ is a Calabi-Yau three fold, and the fluxes $(P, Q)$ go through $S^{2}$ and some three cycles of Calabi-Yau. It was argued that in this case $\psi_{P, Q}(\Phi)$ is the Hartle-Hawking wave function in the Hilbert space $\mathcal{H}_{M}$ obtained by quantization of $H^{3}(M)$ with respect to its symplectic structure.

We now extend this question as follows: Suppose we consider $n$ disconnected copies of $X$, labeled by $X_{i}$, and let the flux $\left(P_{i}, Q_{i}\right)$ pierce through each. Then we ask which state do we get in $\mathcal{H}_{M}^{\otimes n}$ by doing the path integral on geometries whose boundary is $n$ copies of $X$ ? From the geometry (4.18), it is clear that now we get the resulting state

$$
\left|\psi_{n}\right\rangle=\bigotimes_{i=1}^{n}\left|P_{i}, Q_{i}\right\rangle
$$

This is because the states are ground states of the theory and are determined by the long-time evolution, which is precisely the near-horizon geometry of each throat. However we can ask a further refined question: Can this ensemble of baby universes be dual to a single gauge theory? If this were the case there should be tendimensional solutions which connect up all the $X_{i}$ and moreover bound by the constraint that $\sum_{i} P_{i}=P, \sum_{i} Q_{i}=$ $Q$ where the $P, Q$ are fixed by the total flux of the brane where the gauge theory lives. These are precisely the solutions of [22] discussed above. So we would be instructed to write the Hartle-Hawking state as a sum over all allowed fluxes consistent with the fixed total flux and with the constraint that the gravity solutions exist. Let us now see if this expectation agrees with the results for the case we have studied, namely, the case of the $T^{2}$ embedded in the Calabi-Yau. In this case we found (3.18)

$$
\left|\psi_{n}\right\rangle=\sum_{N_{4}^{i}, N_{2}^{i}, N_{0}^{i}} \bigotimes_{i=1}^{n}\left|N_{4}^{i}, N_{2}^{i}, N_{0}^{i}\right\rangle,
$$

$$
\Omega\left(N, N_{2}, N_{0}\right)=\sum_{n=1}^{\infty}(-1)^{n-1} C_{n-1}\left\langle\psi_{n} \mid \psi_{n}\right\rangle,
$$

where each term in the sum is restricted by the condition that

$$
\sum_{i} N_{4}^{i}=N, \quad \sum_{i} N_{2}^{i}=N_{2}, \quad \sum_{i} N_{0}^{i}=N_{0},
$$

and where all $N_{4}^{i}>0$. This is exactly the same structure anticipated in (5.1) from the holographically dual gravity solutions, modulo the factor $(-1)^{n-1}$ in the inner product, which would be interesting to explain from the gravity side. The constraint that $N_{4}^{i}$ all have the same sign is clear from the restriction that the gravity solution exists. Note that, if the sign of $N_{4}$ changes, then there would be points in $\mathbf{R}^{3}$ where the "position-dependent magnetic charges" $P^{I}(x)$, discussed in the last section, all vanish. This is because if we go from a throat with a positive value of $N_{4}$ to one with negative value, we will cross a point where the D4-brane number is zero. Since there are no other magnetic charges in this case, this leads to zero classical entropy for $S(x)$ for some $x$, and thus the gravity solution would become singular.

\section{A. Loss of quantum coherence?}

It is a natural question to ask if our multiuniverse HartleHawking state $\left|\psi_{n}\right\rangle$ leads to a loss of quantum coherence. In a naïve sense one may think that it does, in that we have a sum over all $n$-state wave functions with the total flux condition satisfied. However, as it stands the mixture with the other universes is very simple and captured just by some global flux conservation. In particular if we measure the flux $P_{\text {our }}, Q_{\text {our }}$ in our universe the wave function $\psi_{P_{\text {our }}, Q_{\text {our }}}$ is completely determined. This is consistent with the proposal of Coleman [26] (see also [49-52]) that the creation of baby universes does not lead to a loss of quantum coherence. More precisely it was argued that once one measures the coupling constants in our universe we will have a pure state. This is consistent with our scenario where measuring the flux in our universe is sufficient to lead to a pure state.

\section{B. Lessons for holography}

However, our finding raises a more interesting question in the context of holography: It has been argued that the existence of a unitary gauge theory which is holographically dual to a black hole must automatically lead to a 
resolution of information puzzle for black holes. Here, in the context of our simple example, we are finding that the gauge theory is not dual to a single black hole but to an ensemble of them. In such a scenario the unitarity of the gauge theory evolution operator may not have a direct implication for the unitarity of the physics in a given black hole sector.

There is a precursor for such a sum over geometries being reflected in a gauge theory. This is the case of the finite temperature Yang-Mills theory, where one expects contributions from both the thermal AdS geometry as well as the AdS Schwarzschild geometry. In that case there is, moreover, a phase transition in the semiclassical limit as one varies parameters. This Hawking-Page phase transition of geometries exchanging dominance translates into a large $N$ phase transition in the gauge theory [53]. (See also [5457] for recent studies of this system.)

In the case of the two-dimensional Yang-Mills theory on the torus, while we have seen multiple geometries contributing, we do not have a phase transition as a function of the couplings (or chemical potentials on the gravity side). This is consistent with the expectation on the gravity side as well where the single black hole is always the entropically favored geometry. It would be interesting to consider the issue of scattering off of these extremal black holes and see how the unitarity of the $S$ matrix for the gauge theory is reflected on the gravity side for configurations which can fluctuate to the multicentered black holes.
There are other gravity backgrounds whose holographic dual descriptions involve free fermion Fock spaces; description of black holes in terms of fundamental strings [58-61], Mathur's picture of black hole quantum states [62], BPS states for type IIB string on $\mathrm{AdS}_{5} \times S^{5}[63,64]$, two-dimensional string theory [65-67], etc. It may be possible to extend the description of baby universes we developed in this paper to these and other cases and learn more about nonperturbative phenomena in quantum gravity and string theory.

\section{ACKNOWLEDGMENTS}

We would like to thank A. Adams, M. Aganagic, F. Denef, D. Gaiotto, D. Gross, G. Horowitz, R. Kallosh, M. Kardar, A. Linde, A. Maloney, J. Maldacena, G. Moore, L. Motl, J. Polchinski, J. Preskill, E. Silverstein, A. Strominger, and E. Verlinde for useful discussions. R. D. and H. O. want to thank the Harvard Physics Department for kind hospitality. H.O. also thanks the Institute for Theoretical Physics at the University of Amsterdam for kind hospitality. The research of R. D. was supported by a NWO Spinoza grant and the FOM program String Theory and Quantum Gravity. R. G.'s research has been generously supported by the citizens of India. The research of H.O. was supported in part by DOE Grant No. DE-FG03-92ER40701. The research of C. V. was supported in part by NSF Grants No. PHY-0244821 and No. DMS-0244464.
[1] J. D. Bekenstein, Phys. Rev. D 7, 2333 (1973).

[2] S. W. Hawking, Nature (London) 248, 30 (1974).

[3] A. Strominger and C. Vafa, Phys. Lett. B 379, 99 (1996).

[4] J. M. Maldacena, hep-th/9607235.

[5] A. W. Peet, Classical Quantum Gravity 15, 3291 (1998).

[6] J. R. David, G. Mandal, and S. R. Wadia, Phys. Rep. 369, 549 (2002).

[7] G. Lopes Cardoso, B. de Wit, and T. Mohaupt, Phys. Lett. B 451, 309 (1999).

[8] H. Ooguri, A. Strominger, and C. Vafa, Phys. Rev. D 70, 106007 (2004).

[9] E. Witten, in Proceedings of Salamfestschrift, ICTP, Trieste, 1993, hep-th/9306122.

[10] R. Dijkgraaf, E. Verlinde, and M. Vonk, hep-th/0205281.

[11] M. Bershadsky, S. Cecotti, H. Ooguri, and C. Vafa, Commun. Math. Phys. 165, 311 (1994).

[12] H. Ooguri, C. Vafa, and E. Verlinde, Lett. Math. Phys. 74, 311 (2005).

[13] E. Verlinde, hep-th/0412139.

[14] C. Vafa, hep-th/0406058.

[15] M. Aganagic, H. Ooguri, N. Saulina, and C. Vafa, Nucl. Phys. B715, 304 (2005).

[16] A. Dabholkar, Phys. Rev. Lett. 94, 241301 (2005).

[17] A. Sen, J. High Energy Phys. 05 (2005) 059.
[18] A. Sen, J. High Energy Phys. 07 (2005) 063.

[19] A. Sen, hep-th/0504005.

[20] D. J. Gross and W. I. Taylor, Nucl. Phys. B400, 181 (1993).

[21] A. Dabholkar, F. Denef, G. W. Moore, and B. Pioline, J. High Energy Phys. 08 (2005) 021.

[22] F. Denef, J. High Energy Phys. 08 (2000) 050.

[23] D. Brill, Phys. Rev. D 46, 1560 (1992).

[24] J. M. Maldacena, J. Michelson, and A. Strominger, J. High Energy Phys. 02 (1999) 011.

[25] A. Adams, X. Liu, J. McGreevy, A. Saltman, and E. Silverstein, J. High Energy Phys. 10 (2005) 033.

[26] S. R. Coleman, Nucl. Phys. B307, 867 (1988).

[27] M. R. Douglas, hep-th/9311130.

[28] R. E. Rudd, hep-th/9407176.

[29] R. Dijkgraaf, in The Moduli Space of Curves, Prog. Math., Vol. 129 (Birkhäuser, Boston, Massachusetts, 1995), p. 149.

[30] M. Kaneko and D.B. Zagier, in The Moduli Space of Curves, Prog. Math., Vol. 129 (Birkhäuser, Boston, Massachusetts, 1995), p. 165.

[31] M. Bershadsky, S. Cecotti, H. Ooguri, and C. Vafa, Nucl. Phys. B405, 279 (1993).

[32] R. Dijkgraaf, Nucl. Phys. B493, 588 (1997). 
[33] A. A. Migdal, Sov. Phys. JETP 42, 413 (1975) [Zh. Eksp. Teor. Fiz. 69, 810 (1975)].

[34] B. E. Rusakov, Mod. Phys. Lett. A 5, 693 (1990).

[35] D. S. Fine, Commun. Math. Phys. 134, 273 (1990).

[36] E. Witten, Commun. Math. Phys. 141, 153 (1991).

[37] M. Blau and G. Thompson, Int. J. Mod. Phys. A 7, 3781 (1992).

[38] J. A. Minahan and A. P. Polychronakos, Phys. Lett. B 312, 155 (1993).

[39] M. R. Douglas, hep-th/9303159.

[40] S. Lelli, M. Maggiore, and A. Rissone, Nucl. Phys. B656, 37 (2003).

[41] R. de Mello Koch, A. Jevicki, and S. Ramgoolam, J. High Energy Phys. 08 (2005) 077.

[42] F. J. Dyson, Phys. Rev. 85, 631 (1952).

[43] N. Itzhaki and J. McGreevy, Phys. Rev. D 71, 025003 (2005).

[44] S. Ferrara, R. Kallosh, and A. Strominger, Phys. Rev. D 52, R5412 (1995).

[45] H. Stephani, D. Kramer, M. MacCallum, C. Hoenselaers, and E. Herlt, Exact Solutions to Einstein's Field Equations, Cambridge Monographs on Mathematical Physics (Cambridge University Press, Cambridge, England, 2003), 2nd ed.

[46] D. Brill, Phys. Rev. D 46, 1560 (1992).

[47] B. Bates and F. Denef, hep-th/0304094.

[48] G. W. Gibbons and S. W. Hawking, Phys. Rev. D 15, 2752 (1977).

[49] S. B. Giddings and A. Strominger, Nucl. Phys. B321, 481 (1989).

[50] A. Strominger, "Baby Universes," in Proceedings of TASI
88.

[51] J. Preskill, Nucl. Phys. B323, 141 (1989).

[52] W. Fischler, I. R. Klebanov, J. Polchinski, and L. Susskind, Nucl. Phys. B327, 157 (1989).

[53] E. Witten, Adv. Theor. Math. Phys. 2, 505 (1998).

[54] R. Dijkgraaf, J.M. Maldacena, G. W. Moore, and E. Verlinde, hep-th/0005003.

[55] O. Aharony, J. Marsano, S. Minwalla, K. Papadodimas, and M. Van Raamsdonk, Adv. Theor. Math. Phys. 8, 603 (2004).

[56] O. Aharony, J. Marsano, S. Minwalla, K. Papadodimas, and M. Van Raamsdonk, Phys. Rev. D 71, 125018 (2005).

[57] L. Alvarez-Gaume, C. Gomez, H. Liu, and S. Wadia, Phys. Rev. D 71, 124023 (2005).

[58] A. Dabholkar and J. A. Harvey, Phys. Rev. Lett. 63, 478 (1989).

[59] J. G. Russo and L. Susskind, Nucl. Phys. B437, 611 (1995).

[60] A. Sen, Nucl. Phys. B440, 421 (1995).

[61] A. Sen, Mod. Phys. Lett. A 10, 2081 (1995).

[62] S. D. Mathur, Fortschr. Phys. 53, 793 (2005).

[63] D. Berenstein, J. High Energy Phys. 07 (2004) 018

[64] H. Lin, O. Lunin, and J. Maldacena, J. High Energy Phys. 10 (2004) 025.

[65] J. McGreevy and H. Verlinde, J. High Energy Phys. 12 (2003) 054.

[66] T. Takayanagi and N. Toumbas, J. High Energy Phys. 07 (2003) 064.

[67] M. R. Douglas, I. R. Klebanov, D. Kutasov, J. Maldacena, E. Martinec, and N. Seiberg, hep-th/0307195. 